\title{
A randomized, double-blind, placebo- controlled phase III clinical trial to evaluate the efficacy and safety of SARS-CoV-2 vaccine (inactivated, Vero cell): a structured summary of a study protocol for a randomised controlled trial
}

Murat Akova* ${ }^{*}$ and Serhat Unal

\begin{abstract}
Objectives: The primary objective is to evaluate the efficacy of an inactivated and aluminium hydroxide adsorbed SARS-CoV-2 vaccine (Sinovac, China) in voluntary participants after 14 days of the second dose against RT-PCR confirmed symptomatic COVID-19 cases. The secondary objectives include evaluating the efficacy after at least one dose of the vaccine against RT-PCR confirmed symptomatic COVID-19 cases; the efficacy of two doses of the vaccine on the rates of hospitalization and death; the safety of the vaccine including adverse reactions up to one year after the $2^{\text {nd }}$ dose of vaccination; and the immunogenicity of the vaccine and its duration up to 120 days.

Trial Design: This is a phase III, randomized, double-blind, placebo-controlled case driven clinical trial to assess the efficacy and safety of the vaccine. The study is planned to be carried out within two separate cohorts in voluntary participants aged between 18-59 years old. The first cohort includes healthcare professionals actively working in healthcare units, who are assumed to have higher risk of acquiring COVID-19, and the second cohort includes other immunocompetent subjects in the same age group, who are at a regular risk for COVID-19 disease. In Cohort 1, healthcare professionals will be randomized to receive two intramuscular doses of investigational product or the placebo in a 1:1 ratio and they will be monitored for 12 months by active surveillance of COVID-19. In Cohort 2, immunocompetent subjects will be randomized to receive vaccine or the placebo in a 2:1 ratio.

*Correspondence: makova@hacettepe.edu.tr

Department of Infectious Diseases and Clinical Microbiology, Hacettepe

University School of Medicine, Hacettepe Mh., 06230 Ankara, Turkey

(c) The Author(s). 2021 Open Access This article is licensed under a Creative Commons Attribution 4.0 International License, which permits use, sharing, adaptation, distribution and reproduction in any medium or format, as long as you give appropriate credit to the original author(s) and the source, provide a link to the Creative Commons licence, and indicate if changes were made. The images or other third party material in this article are included in the article's Creative Commons licence, unless indicated otherwise in a credit line to the material. If material is not included in the article's Creative Commons licence and your intended use is not permitted by statutory regulation or exceeds the permitted use, you will need to obtain permission directly from the copyright holder. To view a copy of this licence, visit http://creativecommons.org/licenses/by/4.0/ The Creative Commons Public Domain Dedication waiver (http://creativecommons.org/publicdomain/zero/1.0/) applies to the data made available in this article, unless otherwise stated in a credit line to the data. 
(Continued from previous page)

Participants: Healthcare professionals of both genders, including medical doctors, nurses, cleaners, hospital technicians, and administrative personnel who work in any department of a healthcare unit and immunocompetent individuals of both genders are included. Pregnant (confirmed by positive beta-hCG test) and breastfeeding women as well as those intending to become pregnant within three months after vaccination are excluded. Other exclusion criteria include history of COVID-19 test positivity (PCR or immunoglobulin test results), any form of immunosuppressive therapy including corticosteroids within 6 months, history of bleeding disorders, asplenia, and administration of any form of immunoglobulins or blood products within 3 months.

Exclusion criteria for the second dose include any serious adverse events related with the vaccine, anaphylaxis or hypersensitivity after vaccination, or any confirmed or suspected autoimmune or immunosuppressive disease (including HIV infection).

Participants are only included after signing the voluntary informed consent form, ensuring cooperation in visits, undergoing screening for evaluation, and conforming to all the inclusion and exclusion criteria. All clinical sites are located in Turkey.

Intervention and comparator: The vaccine was manufactured by Sinovac Research \& Development Co., Ltd. It is a preparation made from a novel coronavirus (strain CZ02) grown in the kidney cell cultures (Vero Cell) of the African green monkey and contains inactivated SARS-CoV-2 virus, aluminium hydroxide, disodium hydrogen phosphate, sodium dihydrogen phosphate, and sodium chloride. A dose of $0.5 \mathrm{~mL}$ contains 600 SU of SARS-CoV-2 virus antigen. The placebo contains aluminium hydroxide, disodium hydrogen phosphate, sodium dihydrogen phosphate, and sodium chloride $(0.5 \mathrm{~mL} /$ dose $)$.

Scheduled visits and additional unscheduled weekly visits will be performed for the first 13 weeks and neutralizing antibody test, IgG test, T-Cell activation test, pregnancy test, and RT-PCR tests along with total antibody test will be performed. Adverse events and serious adverse events during the follow-up will be recorded on diary cards. Diary cards will collect information on the timing and severity of COVID-19 symptoms and solicited adverse events recorded by the subjects during one-year follow-up period. All serious adverse events will be managed and necessary treatment will be ensured according to the local regulations. All serious adverse events following vaccination will be reported to the ethics committee, the Ministry of Health, and the study sponsor within 24 hours of detection.

Main Outcomes: The primary efficacy endpoint is the incidence of symptomatic cases of COVID-19 disease confirmed by RT-PCR two weeks after the second dose of vaccination. Secondary efficacy endpoints are the incidence of hospitalization/mortality rates among one or two dose regimens, duration of immunogenicity rates up to 120 days, the seroconversion rate, the seropositivity rate, neutralizing antibody titer, and lgG levels 14 days after each dose of vaccination. The primary safety endpoint is the severity and frequency of local and systemic adverse reactions during the period of one week after vaccination. The study would be terminated if more than $15 \%$ of the subjects have grade $\geq 3$ adverse events related to vaccination including local reactions.

Randomisation: Eligible subjects will be randomized at their Study Day 0 to two study groups using an Interactive Web Response System (IWRS; developed by Omega CRO, Ankara, Turkey) in both risk groups. The IWRS system customizes the randomization algorithm. After enrolment in the study, each participant will be randomly assigned to either of the two treatment arms at a ratio of 1:1 in the high-risk group and at a ratio of 2:1 in the normal risk group. Each enrolled participant will be assigned to a code and will receive the treatment labelled with the code.

Blinding (masking): The trial is a double-blind study to avoid introducing bias. The blinding may be broken by the investigator in the event of a medical emergency in which knowledge of the identity of the study vaccine is critical for management of the subject's immediate treatment. The Data and Safety Monitoring Board is to be contacted in case of breaking the blinding for a study object. The blood samples will be taken from both placebo and vaccinated groups, in order not to break the blinding.

(Continued on next page) 
(Continued from previous page)

Numbers to be randomised (sample size): The study is planned to be carried out with two separate cohorts. The Cohort 1 includes healthcare professionals working in healthcare units and the Cohort 2 consists of immunocompetent subjects having normal risk for COVID-19 disease. The Cohort 2 will be initiated after the evaluation of the interim safety report of the Cohort 1 by the Data and Safety Monitoring Board. Both cohorts will be followed-up via RT-PCR to confirm symptomatic COVID-19 cases. If the clinical efficacy of the vaccine is shown in the Cohort 1 or 2, the subjects randomized into the placebo arm will also be vaccinated. In the Cohort 1, 588 subjects should be included in both arms with the assumption that the risk of infection with COVID-19 will be 5\% for the placebo arm and 2\% for the vaccine arm in the high-risk group. Considering 10\% of drop-out rate and 5\% of seropositivity or PCR positivity at baseline, 680 subjects should be screened at both arms of the Cohort 1. Group sample sizes of 7545 SARS-CoV-2 vaccine and 3773 placebo suits at a two-sided 95\% confidence interval for the difference in population proportions with a width equal to $1.0 \%$, when the estimated incidence rate for vaccinated group is $1.0 \%$ and the estimated incidence rate for placebo group is $2.0 \%$. Drop-out rate is assumed to be $10 \%$ and seropositivity or PCR positivity at baseline is assumed to be 5\%; accordingly, 13000 participants are needed to be enrolled totally in both cohorts. The remaining 11640 subjects will be screened in the Cohort 2 and eligible subjects will be randomized at a ratio of 2:1.

Trial Status: Protocol version 6.0 - 15 October 2020. Recruitment started on 15.09 .2020 and is expected to end on February 2022.

Trial Registration: ClinicalTrials.gov, NCT04582344. Registered 8 October 2020

Full Protocol: The full protocol of the trial is attached as an additional file, accessible from the Trials website (Additional file 1). In the interest in expediting dissemination of this material, the familiar formatting has been eliminated; this Letter serves as a summary of the key elements of the full protocol.

Keywords: COVID-19, randomised placebo controlled trial, double blind, protocol, inactivated vaccines, phase III clinical trial, COVID-19, vaccine, Turkey

\section{Supplementary Information}

The online version contains supplementary material available at https://doi. org/10.1186/s13063-021-05180-1.

Additional file 1. Full Study Protocol

\section{Acknowledgements}

The authors thank Omega CRO, Ankara, Turkey for providing support for the site management and trial organization. Omega also acts as the CRO representing the Turkish Health Institutes Association (TUSEB) during the trials.

\section{Authors' contributions}

Both authors conceived and designed the trial and wrote the trial protocol. Both authors approved the final version of the protocol.

\section{Funding}

The study was funded by the Turkish Health Institutes Association (TUSEB) in the design of the study, in collection, analysis, and interpretation of data, and in writing the manuscript. Synovac provided the vials of vaccine and the placebo for the trial to be used in volunteers.

\section{Availability of data and materials}

Not applicable.

\section{Declarations}

\section{Ethics approval and consent to participate}

This study was reviewed and approved by the Clinical Investigations Ethics Committee of Hacettepe University (No: 2020/10-26 and Date: 16.07.2020).

Signed informed consents of all participants will be obtained.
Competing interests

The authors declare that they have no competing interests.

Received: 5 March 2021 Accepted: 10 March 2021

Published online: 13 April 2021

\section{Publisher's Note}

Springer Nature remains neutral with regard to jurisdictional claims in published maps and institutional affiliations.
Ready to submit your research? Choose BMC and benefit from:

- fast, convenient online submission

- thorough peer review by experienced researchers in your field

- rapid publication on acceptance

- support for research data, including large and complex data types

- gold Open Access which fosters wider collaboration and increased citations

- maximum visibility for your research: over $100 \mathrm{M}$ website views per year

At BMC, research is always in progress.

Learn more biomedcentral.com/submissions 